behavior of the disaccharidases in our experimental model remains a moot point.

The absence of morpohologic changes by light microscopy in our model probably is due to the short time which elapsed after challenge. Using a similar model, Perdue et al. (unpublished data) found that while morphologic changes could not be identified $1 \frac{1 / 2}{h}$ after challenge, after $4 \mathrm{~h}$, edema and shredding of enterocytes could be demonstrated.

\section{REFERENCES}

1. Kuitunen P, Visakorpi JK, Hallman N 1965 Histopathology of duodenal mucosa in the malabsorption syndrome induced by cow's milk. Ann Paediatr 205:54-61

2. Loeb H, Vanisel M, Cadranel S, Jacky A, Diederick M, Walter R 1971 Malabsorption and villous atrophy in four infants: relationship to cow's milk intolerance. Acta Paediatr Scand 60:372-380

3. Kletter B, Gery I, Freier S, Noah Z, Davies AM 1971 Immunoglobulin E antibodies to milk proteins. Clin Allergy 1:249-255

4. Weil S, Kuperman O, Ilfeld D, Finelt M, Freier S 1982 Non-specific suppressor cell activity and lymphocyte response to beta-lactoglobulin in cow milk hypersensitivity. J Pediat Gastroenterol Nutr 1:389-393

5. Byars N, Ferraresi RW 1976 Intestinal anaphylaxis in the rat as a model of food allergy. Clin Exp Immunol 24:352-356
6. Bloom G, Kelly JW 1960 The copper phthalocyamine dye Astrablau and its staining properties, especially the staining of mast cells. Histochemie 2:4857

7. Dahlquist A 1968 Assay of intestinal disaccharidases. Anal Biochem 22:99 107

8. Goldstein R, Klein T, Freier S, Menczel J 1970 Alkaline phosphatase and disaccharidase activities in the rat intestine from birth to weaning. I Effect of diet on enzyme development. Am J Clin Nutr 24:1224-1231

9. Pesce MA, Strande CS 1973 A new micromethod for determination of protein in cerebrospinal fluid and urine. Clin Chem 19:1265-1268

10. Jarrett EEE 1978 Stimuli for the production and control of IgE in rats. Immunol Rev 41:52-76

11. Belut D, Moneret-Vantrin DA, Nicolas JP, Grilliat JP 1980 IgE levels in intestinal juice. Dig Dis Sci 25:323-328

12. Iyngkaran N, Davis K, Robinson MJ, Boey CG, Sumithran E, Yadav M, Lam SK, Puthucheary SD 1979 Cow's milk protein sensitive enteropathy: an important contributing cause of secondary sugar intolerance in young infants with acute infective enteritis. Arch Dis Child 54:39-43

13. Perdue MH, Chung M, Gall DG 1984 Effect of intestinal anaphylaxis on gut function in the rat. Gastroenterology 86:391-397

14. Cezard JP, Conklin KA, Das BC, Gray GM 1979 Incomplete intra-cellular forms of intestinal surface membrane sucrase-isomaltase. J Biol Chem 254:8969

15. Saito M, Murakami E, Suda M 1976 Circadian rhythms in disaccharidases of rat small intestine and its relation to food intake. Biochem Biophys Acta 421:177-179

\title{
Diagnostic and Therapeutic Implications of Medium-Chain Acylcarnitines in the Medium-Chain Acyl-CoA Dehydrogenase Deficiency
}

\author{
CHARLES R. ROE, DAVID S. MILLINGTON, DAVID A. MALTBY, TIMOTHY P. BOHAN, \\ STEPHEN G. KAHLER, AND RONALD A. CHALMERS
}

Division of Genetics and Metabolism, Department of Pediatrics, Duke University Medical Center, Durham, North Carolina 27710 [C.R.R., D.S.M., D.A.M., T.P.B., S.G.K.], and Section of Perinatal and Child Health, Clinical Research Centre, Harrow, Middlesex, England [R.A.C.]

\begin{abstract}
The medium-chain acyl-coA dehydrogenase deficiency is one of several metabolic disorders presenting clinically as Reye syndrome. Evidence is presented for a characteristic organic aciduria that distinguishes this disorder from Reye syndrome and other masqueraders characterized by dicarboxylic aciduria. The key metabolites, suberylglycine and hexanoylglycine, are excreted in high concentration only when the patients are acutely ill. More significantly, using novel techniques in mass spectrometry, the medium-chain defect is shown to be characterized by excretion of specific medium-chain acylcarnitines, mostly octanoylcarnitine, without significant excretion of a normal
\end{abstract}

Received August 30, 1984; accepted December 31, 1984.

Supported in part by a grant from the National Reye's Syndrome Foundation, Bryan, OH, the Reye Syndrome Research Fund-SFA (Duke University Medical Center), and the RR-30 General Clinical Research Center Program Division of Research Resources, NIH, Bethesda, MD.

Correspondence to Dr. David S. Millington, Division of Genetics and Metabolism, Department of Pediatrics, Duke University Medical Center, Durham, NC 27710 . metabolite, acetylcarnitine, in four patients with documented enzyme deficiency. Similar studies on the urine of two patients reported with Reye-like syndromes of unidentified etiology have suggested the retrospective diagnosis of medium-chain acyl-coA dehydrogenase deficiency. Administration of $L$-carnitine to medium-chain acyl-coA dehydrogenase deficiency patients resulted in the enhanced excretion of medium-chain acylcarnitines. Octanoylcarnitine is prominent in the urine both prior to and following L-carnitine supplementation. The detection of this metabolite as liberated octanoic acid, following ion-exchange chromatographic purification and mild alkaline hydrolysis, provides a straightforward diagnostic procedure for recognition of this disorder without subjecting patients to the significant risk of fasting. In view of the carnitine deficiency and the demonstrated ability to excrete the toxic mediumchain acyl-coA compounds as acylcarnitines, a combined therapy of reduced dietary fat and $\mathrm{L}$-carnitine supplementation $(25 \mathrm{mg} / \mathrm{kg} / 6 \mathrm{~h})$ has been devised and applied with 
positive outcome in two new cases. (Pediatr Res 19: 459466, 1985)

\section{Abbreviations}

MCAD, medium-chain acyl-coA dehydrogenase deficiency coA, coenzyme A

GC/MS, gas chromatography-mass spectrometry

FAB, fast atom bombardment

$B$, magnetic field

E, electric field

Several inherited metabolic diseases including propionic acidemia, methylmalonic aciduria, isovaleric acidemia, hydroxymethylglutaric aciduria, multiple acyl-coA dehydrogenase deficiency, and more recently, MCAD are occasionally confused with Reye syndrome owing to similar clinical presentation. During acute illness, these disorders frequently have an associated dicarboxylic aciduria, with low free carnitine and relatively increased short-chain acylcarnitines in both urine and plasma. Although dicarboxylic aciduria is a frequent concomitant of reduced carnitine, there is growing evidence that the acylcarnitines excreted in these disorders are derived from metabolites characteristic of the enzyme deficiency (1).

MCAD is an intramitochondrial defect in fatty acid oxidation associated with reduced plasma and tissue carnitine levels (2). This disorder presents with life-threatening hypoglycemia and coma reminiscent of Reye syndrome. Deaths have been reported in affected siblings whose postmortem findings included fat accumulation in hepatocytes, brain swelling, and uncal herniation (2). Recently developed assays for the dehydrogenase enzyme activity summarized by Gregerson (3) have confirmed MCAD in approximately 10 cases $(2,4-8)$. Numerous other cases have been reported which are very similar in clinical description but without enzymatic verification, where MCAD is indicated by the excretion of metabolites consistent with the disorder $(9-18)$. Such patients, presenting with recurrent hypoglycemia of unknown etiology, are frequently subjected to a diagnostic fast to assess the integrity of gluconeogenesis during fasting stress. In some instances this can be disastrous, resulting in death (9) or coma responding to glucose infusion (19). It is not yet clear whether glucose deficiency or an excess of other metabolites is responsible for clinical deterioration in this disease. Similarly, there has been no explanation for the abnormality in carnitine metabolism associated with this disorder.

This report describes two additional families with three affected children. Studies on these children are compared with results obtained from patients with Reye syndrome, and patients with dicarboxylic aciduria secondary to dietary load. In addition, urine samples from the patients reported earlier by Stanley $e t a l$. (2), Gregerson et al. (9), and by Chalmers et al. (11) were studied for comparison.

The studies describe the distinguishing organic acid metabolites in these disorders in addition to the characteristic acylcarnitines which were identified using novel mass spectrometric techniques. The data indicate that MCAD can be readily recognized by metabolite analysis without subjecting patients to the risk of diagnostic fasting and may be treated effectively using supplemental L-carnitine.

\section{MATERIALS AND METHODS}

Octanoylcarnitine and hexanoylcarnitine were synthesized, purified, and characterized by methods previously described ( 20 , 21). Hexanoylglycine and suberylglycine were synthesized by conjugation of the corresponding acid chloride with glycine under basic conditions. Water was deionized and distilled; all solvents and chemicals were of reagent grade.
Plasma and urinary free carnitine, short-chain acylcarnitines, and long-chain acylcarnitines were determined by the radioenzymatic assay utilizing $\left[{ }^{14} \mathrm{C}-1\right]$ acetyl-coA and carnitine acetyltransferase as described by Brass and Hoppel (22).

To obtain organic acid profiles, urine samples $(2 \mathrm{ml})$ were treated with ethoxyamine hydrochloride $(0.4 \mathrm{~g}$, Fisher Scientific, Raleigh, NC) acidified to $\mathrm{pH} 1$, saturated with sodium chloride, then successively extracted with ether $(3 \times 2 \mathrm{ml})$ and ethyl acetate $(3 \times 2 \mathrm{ml})$. The combined extracts were evaporated under a stream of nitrogen, derivatized with bis(trimethylsilyl) trifluroacetamide (Sigma Chemical Co., St. Louis, MO), and analyzed by $\mathrm{GC} / \mathrm{MS}$. The samples $(2 \mu \mathrm{l})$ were injected (temperature $250^{\circ} \mathrm{C}$, split ratio 1:50) onto a $30 \mathrm{~m} \times 0.25 \mathrm{~mm}$ id fused silica capillary GC column (DB-1, J \& W Scientific, Rancho Cordova, CA) directly coupled to the ion source of the HP-5985 gas chromatograph-mass spectrometer (Hewlett-Packard, Inc., Palo Alto, CA). After an initial hold at $120^{\circ} \mathrm{C}$ for $5 \mathrm{~min}$, the oven temperature was programmed at $8^{\circ} \mathrm{C} / \mathrm{min}$ to $280^{\circ} \mathrm{C}$. The MS was scanned repetitively over the mass range 500-50 at 800 daltons/ $\mathrm{s}$ and all data stored on cartridge disks compatible with the HP7920 disk drive. The ion source was operated in EI mode at $200^{\circ}$ $\mathrm{C}$ with an electron energy of $70 \mathrm{eV}$.

For the identification of acylcarnitines, each of the urine samples $(0.5-2.0 \mathrm{ml})$ was acidified and passed through a column $(30 \mathrm{~mm} \times 4 \mathrm{~mm}$ ) of Dowex-50 ion-exchange resin (Bio-Rad Laboratories, Richmond, CA). After washing with $0.01 \mathrm{~N} \mathrm{HCl}$ $(15 \mathrm{ml})$ and water $(15 \mathrm{ml})$, the acylcarnitines were eluted with 1 $\mathrm{N} \mathrm{NH}_{4} \mathrm{OH}$ in ethanol/water (1:4) and the mixture lyophilized. The residue was treated with urease $(1$ unit, Sigma Chemical Company) in water $(1.0 \mathrm{ml})$ at $37^{\circ} \mathrm{C}$ for $0.5 \mathrm{~h}$, then lyophilized again. The residue was then dissolved in $0.01 \mathrm{~N} \mathrm{HCl}(0.2 \mathrm{ml})$ prior to analysis by fast atom bombardment-MS and constant B/E ratio linked scanning. These analyses were performed on 2$5 \mu \mathrm{l}$ of sample using a VG-7070HS/11-250 (VG-Instruments, Inc., Stamford, CT) mass spectrometer/datasystem combination as described earlier (21). The remaining sample was hydrolyzed $\left(0.2 \mathrm{~N} \mathrm{KOH}, 37^{\circ} \mathrm{C}, 1 \mathrm{~h}\right)$, acidified to $\mathrm{pH} 2$, and extracted with ether $(3 \times 1.0 \mathrm{ml})$. The combined extract was treated with excess ethereal diazomethane and carefully concentrated under $\mathrm{N}_{2}$ to a final volume of about $0.1 \mathrm{ml}$, then analyzed by capillary GC/ MS.

\section{CASE REPORTS}

Patients with Reye syndrome. Three patients presenting with Reye syndrome in stage II are included in this analysis. These same patients were studied again 8-12 months following that episode and are considered control patients at that time.

Patients on ketogenic diet. Three additional patients with dicarboxylic aciduria due to excessive medium-chain triglyceride intake in the diet are also included for study of the specificity of urinary organic acid and acylcarnitine analyses compared to children with MCAD deficiency or Reye syndrome.

Patient $R-1$. A female patient presented to Duke Medical Center at 13 months of age, $1 \mathrm{wk}$ following hospitalization for suspected Reye syndrome. She had a respiratory infection with fever which progressed to lethargy and unresponsiveness following a high fat meal. She had two generalized seizures associated with a plasma glucose of $22 \mathrm{mg} / \mathrm{dl}$, CSF glucose 7, ammonia 112 $\mu \mathrm{g} / \mathrm{dl}$, normal sodium and potassium, $\mathrm{CO}_{2} 17$, SGOT $224 \mathrm{IU} /$ L. CT scan on admission suggested petechial hemorrhages in the right fronto-parietal area. Physical examination revealed a febrile, flaccid, unresponsive female with hepatomegaly $(5 \mathrm{~cm})$, but who appeared normal in weight and height. Developmental history was normal. Her neurological exam was improved by $48 \mathrm{~h}$ and she was awake and responsive by the 6th hospital day. Analysis of urinary organic acids during this interval revealed dicarboxylic aciduria, 5-hydroxyhexanoate, hexanoylglycine, suberylglycine, and octanoate. Plasma and urinary carnitine data are presented in Table 1 as baseline values. The association of the organic aciduria with low plasma and urine free carnitine prompted 
Table 1. Effect of oral carnitine load $(100 \mathrm{mg} / \mathrm{kg}$ ) on plasma and urinary carnitine levels* in MCAD

\begin{tabular}{|c|c|c|c|}
\hline & & Free & SC-acyl \\
\hline \multicolumn{4}{|l|}{ Plasma } \\
\hline \multicolumn{4}{|l|}{ A. Baseline } \\
\hline \multirow[t]{2}{*}{ Patients: } & R-1 & 8.3 & 2.0 \\
\hline & K & 22.2 & 7.3 \\
\hline \multirow[t]{2}{*}{ Controls: } & $(n=3)$ & 32.3 & 3.5 \\
\hline & (Range) & $(31.2-33.3)$ & $(2.6-4.2)$ \\
\hline \multicolumn{4}{|c|}{ B. Post carnitine $\dagger$} \\
\hline \multirow[t]{2}{*}{ Patients: } & R-1 & 50.0 & 9.0 \\
\hline & $\mathrm{K}$ & 69.0 & 12.4 \\
\hline \multirow[t]{3}{*}{ Controls: } & $(n=3)$ & 66.3 & 31.6 \\
\hline & (Range) & $(50-85)$ & $(11.2-57)$ \\
\hline & & Free & Acyl $\ddagger$ \\
\hline \multicolumn{4}{|l|}{ Urine } \\
\hline \multicolumn{4}{|l|}{ A. Baseline } \\
\hline \multirow[t]{2}{*}{ Patients: } & R-1 & 4 & 56 \\
\hline & $\mathrm{K}$ & 54 & 165 \\
\hline \multirow[t]{2}{*}{ Controls: } & $(n=3)$ & 24 & 103 \\
\hline & (Range) & $(13-34)$ & $(61-160)$ \\
\hline \multicolumn{4}{|c|}{ B. Post carnitine $\dagger$} \\
\hline \multirow[t]{2}{*}{ Patients: } & $R-1$ & 1385 & 563 \\
\hline & $\mathrm{K}$ & 2024 & 669 \\
\hline \multirow[t]{2}{*}{ Controls: } & $(n=3)$ & 6448 & 2129 \\
\hline & (Range) & $(4517-8252)$ & $(483-3069)$ \\
\hline
\end{tabular}

* Carnitine expressed as $\mathrm{nmol} / \mathrm{ml}$ of plasma and $\mathrm{nmol} / \mathrm{mg}$ creatinine in urine. SC-acyl, short- and medium-chain acylcarnitines.

$\dagger$ Specimens collected at the peak of acylcarnitine excretion, usually 6-8 $\mathrm{h}$ after the load.

$\$$ Acyl, combined acylcarnitines. Approximately $63 \%$ of these values for the patients $\mathrm{R}-1$ and $\mathrm{K}$ is accounted for by octanoylcarnitine (see text).

reduction of dietary fat $(<20 \%$ total $\mathrm{kcal} /$ day $)$ and $\mathrm{L}$-carnitine supplementation $(25 \mathrm{mg} / \mathrm{kg} / 6 \mathrm{~h})$. This regimen resulted in an improved urinary organic acid profile, restoration of normal free carnitine levels, reduced hepatic enlargement, and normal serum transaminases.

Her course since discharge has been complicated by complex partial seizures without hypoglycemia or organic aciduria but controlled by phenobarbital. At age $25 / 12$ yr, she is developmentally and intellectually normal. Her parents are unrelated and normal (carnitine status and GC-MS of organic acids).

Patient $R-2$. This male sibling of patient $\mathrm{R}-1$ was a normal product of a term pregnancy. He had no prior illness when at 7 months of age he was found dead in his crib in the early morning hours. Autopsy revealed marked fatty change of the liver, diffuse cerebral edema, marked acute interstitial pneumonia, and myocarditis apparently due to Coxsackie B-5 viral infection. Biological fluids and tissue were not available for study.

Patient $K$. The patient was well until 7 months of age when she developed bilateral serous otitis media. After 2 days of therapy, she was found unconscious, limp, and had jerking eye movements. She was hospitalized and found to have a blood glucose level of $4 \mathrm{mg} / \mathrm{dl}$. At 11 months of age, she was again found limp, clammy, and unconscious. Blood glucose at that time was $30 \mathrm{mg} / \mathrm{dl}$. She has had seven additional episodes of hypoglycemia. She was admitted to Duke Medical Center where urinary organic acid analysis by GC-MS revealed the same metabolites as in patient R-1. The plasma and urinary carnitine concentrations are given in Table 1 (baseline data). Dietary fat was restricted and she improved markedly. She has since been treated with L-carnitine $25 \mathrm{mg} / \mathrm{kg} / 6 \mathrm{~h}$ and has experienced no further hypoglycemic episodes or hospitalization. Her parents are unrelated and there is no family history of hypoglycemia, coma, or death in childhood.

\section{RESULTS}

Enzyme assays. Fibroblast cultures from patients R-1 and $\mathrm{K}$ were assayed for long-, medium-, and short-chain acyl-coA dehydrogenase activity, using palmityl-coA, octanoyl-coA, and butyryl-coA respectively as the substrates. The assays were performed at the Children's Hospital, Philadelphia by Dr. P. Coates, using a method (23) similar to that described by Frerman et al. (24). The values obtained, expressed as nmol ETF reduced/min/ $\mathrm{mg}$ protein, are as follows. R-1: $1.99,0.22,1.18 ; \mathrm{K}: 2.14,0.29$, 0.88 ; normals: $2.24 \pm 0.26(n=14), 3.79 \pm 0.57(n=14), 2.24$ $\pm 0.22(n=5)$.

Organic acid analyses. Urinary organic acid analysis by GCMS revealed specific differences between Reye syndrome and MCAD. In Figure $1 A$, the total ion current chromatogram of an
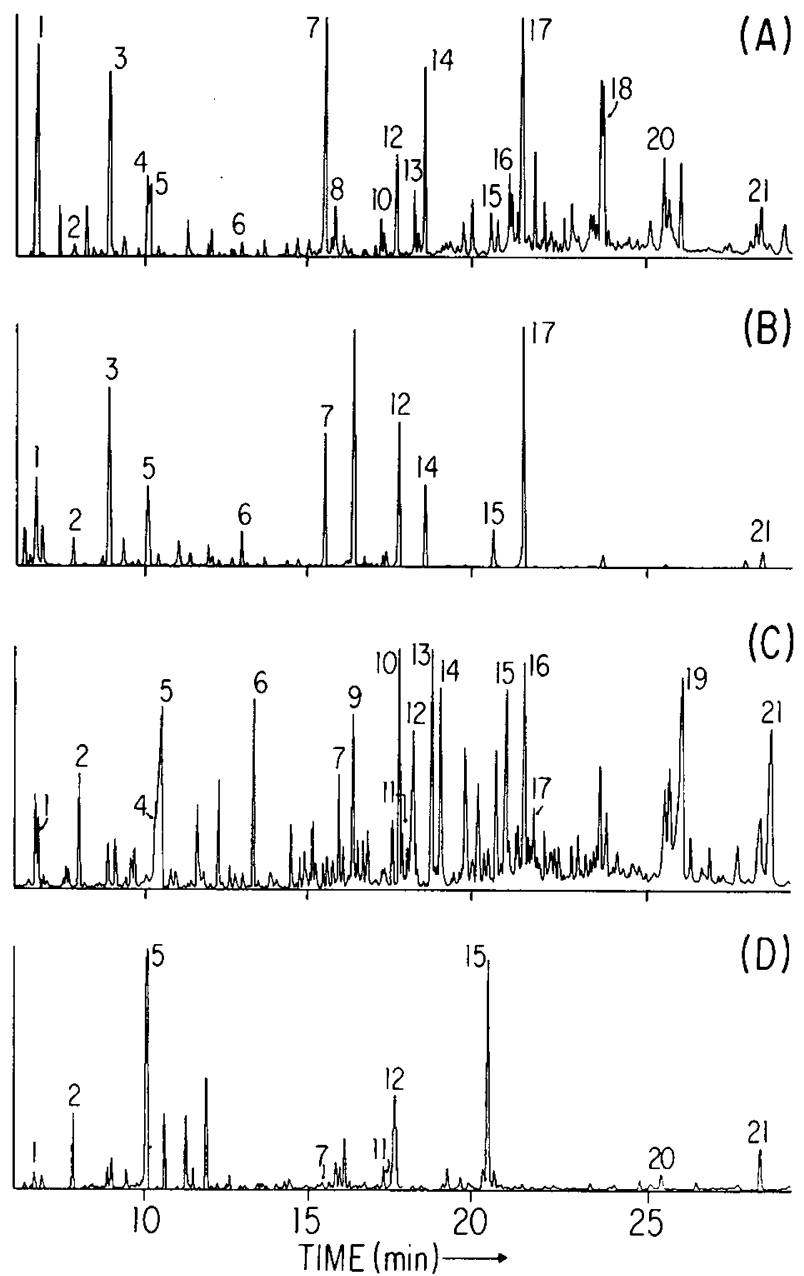

Fig. 1. Urinary organic acid profiles obtained by capillary column GC/MS. $A$, Reye's syndrome (acute)-characterized by elevated lactate, ketones, saturated and unsaturated dicarboxylic acids, salicylate metabolites. $B$, excessive dietary MCT-elevated lactate, ketones, saturated dicarboxylic acids. $C$, MCAD when sick-shows no ketones, elevated 5hydroxyhexanoic, saturated and unsaturated dicarboxylic acids, hexanoylglycine, and suberylglycine. $D, \mathrm{MCAD}$ when well—only abnormality is a small peak of hexanoylglycine (peak 11). Key: 1, lactic; 2, oxalic; 3, 3-hydroxybutyric; 4, 3-hydroxyisovaleric; 5, urea; 6, 5-hydroxyhexanoic; 7 , adipic; 8 , salicylic; 9, dehydroadipic; 10, 2-oxoglutaric; 11 , hexanoylglycine; 12, 4-hydroxyphenylacetic; 13 , dehydrosuberic; 14 , suberic; 15 , hippuric; 16 , dehydrosebacic; 17 , sebacic; 18 , salicyluric; 19 , suberylglycine; 20, 4-hydroxyhippuric; $21, \mathrm{C}_{24} \mathrm{H}_{50}$. Note: these analyses were performed at different times and under slightly different conditions, hence the elution times for each component are not exactly the same. Components were identified by matching GC retention and mass spectrum with those of authentic standards, except for unsaturated dicarboxylic acids for which standards were unavailable. 
admission urine sample from a child with stage II Reye syndrome is shown. The major features include dicarboxylic aciduria (adipate, suberate, sebacate), a small amount of 5-hydroxyhexanoate, aspirin metabolites (salicylate, gentisate, and salicylurate), significant ketosis (3-OH butyrate, acetoacetate), and greatly increased lactate. The only glycine conjugates present are hippurate (benzoylglycine) and salicylurate (salicylglycine).

Organic acids in three patients with dicarboxylic aciduria secondary to increased medium chain triglyceride intake were also studied by GC-MS. A typical profile is presented in Figure $1 B$. The usual dicarboxylic acids, adipate, suberate, and sebacate were noted along with 5-hydroxy hexanoate. No acylglycines other than hippurate or unsaturated dicarboxylic acids were noted.

Figure $1 C$ shows the organic acid profile of patient $R-1$ during the Reye-like episode. The major distinguishing features seen in this example of MCAD are as follows: dicarboxylic aciduria including saturated and unsaturated medium-chain dicarboxylic acids, 5-hydroxyhexanoate, hexanoylglycine, suberylglycine, and low ketones. When this patient was well, only a small amount of hexanoylglycine was noted (Fig. $1 D$ ) in an otherwise normal profile.

A similar analysis was performed on samples from patient TB previously reported as having a Reye-like illness in which 5-OH hexanoate was first identified by Chalmers et al. (11). Adipic, suberic, sebacic, octenedioic, decenedioic, and octanoic acids were also reported. The new studies of this patient's urine clearly reveal significant peaks of both hexanoylglycine and suberylglycine, standards of which were not available to Chalmers et al. (11) at the time of their study. The organic acid profiles were very similar to the one shown in Figure $1 C$. The GC/MS analysis of samples from patients $\mathrm{M}-1$ and $\mathrm{B}-2$ with documented MCAD, recently described by Stanley et al. (2), also reveal hexanoylglycine and suberylglycine. Similarly, urine from patient $\mathrm{JG}$, in which Gregerson et al. (9) recognized suberylglycine as the methyl ester was shown to contain all the metabolites described for the MCAD patients including hexanoylglycine and free octanoate.

In Figure 2, the mass spectra of the TMS derivatives of hexanoylglycine and suberylglycine and of hexenedioic, octenedioic, and decenedioic acids (dehydro-adipic, -suberic, -sebacic acids) are presented. These are useful in the recognition of the medium-chain defect. To our knowledge, only the mass spectrum of suberylglycine-TMS derivative has been previously published $(25,26)$.

Carnitine supplementation. The two patients with MCAD and three control patients were each given an oral L-carnitine load of $100 \mathrm{mg} / \mathrm{kg}$. Prior to carnitine administration, the plasma and
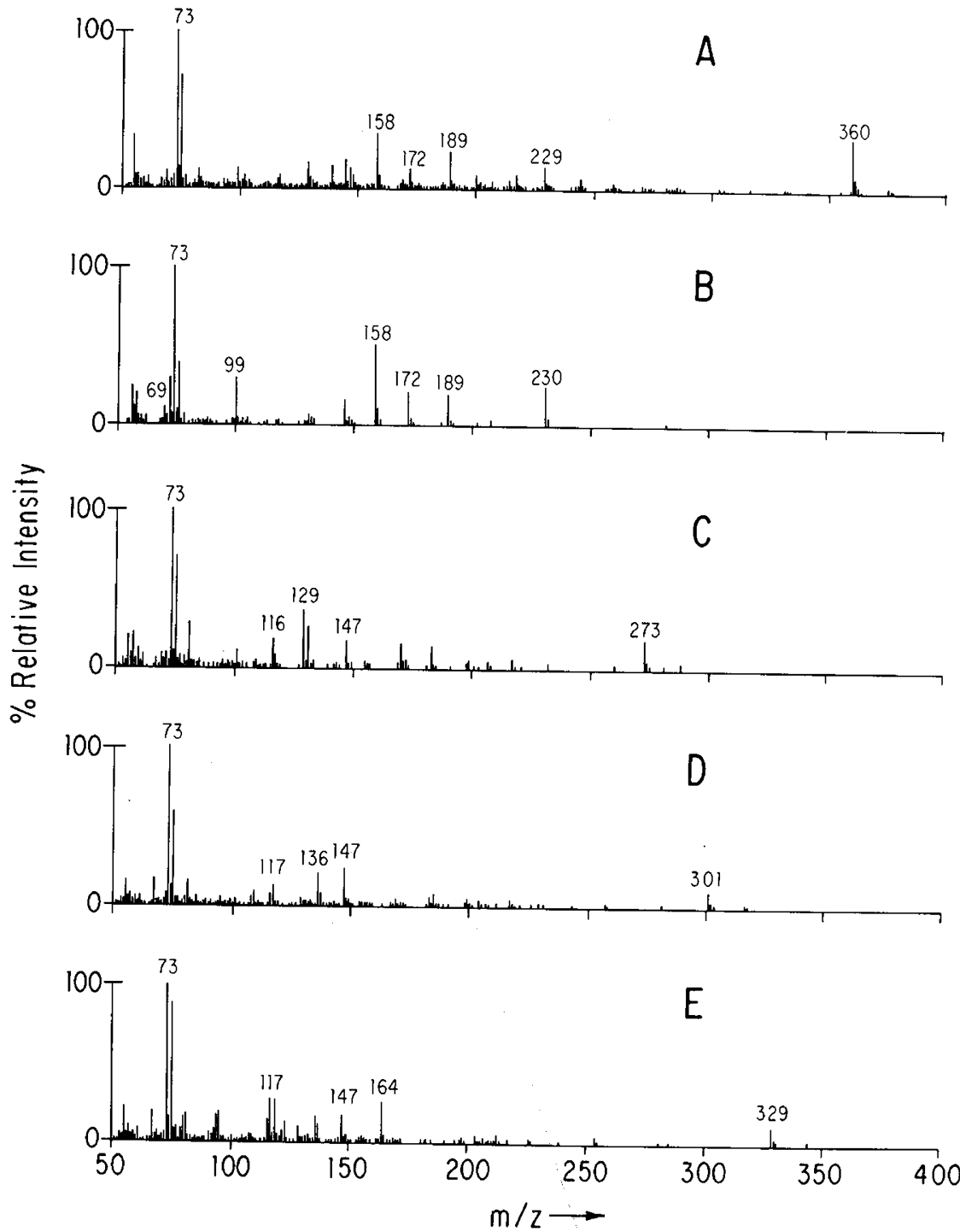

Fig. 2. Mass spectra (EI) from TMS derivatives of urinary metabolites characteristic of MCAD when patient is symptomatic. $A$, suberylglycine; $B$, hexanoylglycine; $C$, hexenedioic acid; $D$, octenedioic acid; $E$, decenedioic acid. 

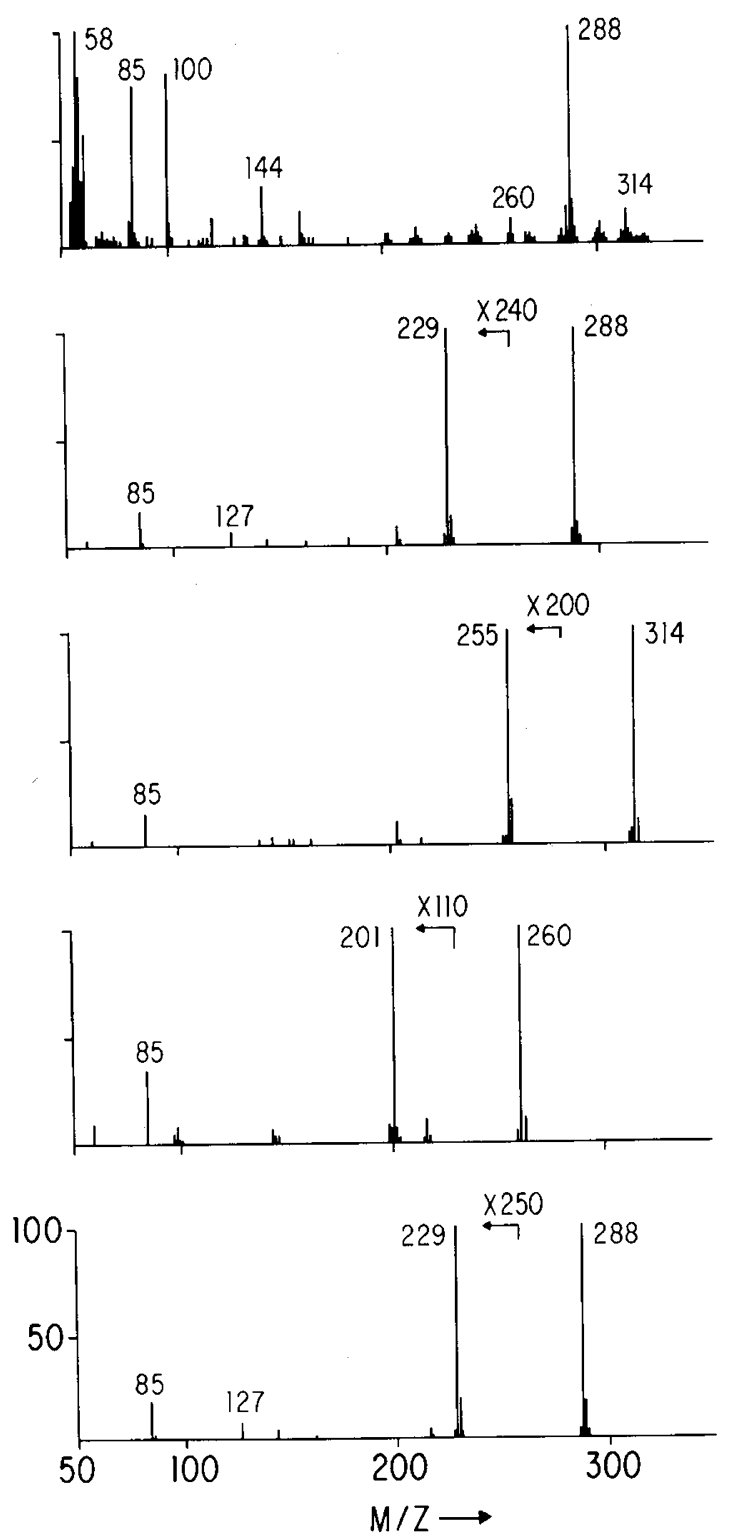

Fig. 3. Analysis of acylcarnitines in MCAD urine by FAB-MS and constant $\mathrm{B} / \mathrm{E}$ ratio linked scanning. $A, \mathrm{FAB}-\mathrm{MS}$ spectrum from purified urine of patient R-1 after carnitine administration, showing dominant signals from octanoylcarnitine $(\mathrm{m} / \mathrm{z} 288,144,100,85,58)$ and its homologs. No prominent signal is observed for acetylcarnitine $(\mathrm{m} / \mathrm{z} 204)$. $B-D$, fragmentation patterns of $\mathrm{m} / \mathrm{z} 288,314$, and 260 ions in patient urine obtained by constant B/E ratio linked scan. Signals at $(\mathrm{MH}-59)^{+}$ and $\mathrm{m} / \mathrm{z} 85$ are characteristic of acylcarnitines. $E$, fragmentation pattern of synthetic octanoylcarnitine $\mathrm{MH}^{+}$ion is identical with that of the $\mathrm{m} / \mathrm{z}$ 288 ion in the patient's urine.

urinary free carnitine levels in patient $\mathrm{R}-1$ were significantly lower than the control values (Table 1). Patient $\mathrm{K}$ had only a slight decrease in plasma free carnitine and normal urinary free carnitine, but was already on a diet restricted in fat, in contrast to patient R-1.

Following carnitine supplementation, plasma and urinary levels of all carnitine fractions increased in all patients. The maximum excretion occurred approximately $6 \mathrm{~h}$ after the bolus was administered. Plasma free carnitine was normalized in the MCAD patients and, as expected, increased in the controls. The increase in short-chain acylcarnitines in plasma and urine was much greater in the controls than in the patients. Similarly, the concentration of urinary free carnitine was much greater in the controls than the patients, even though each received the same oral dose. Although the proportion of acylcarnitines excreted relative to total carnitine $(25-29 \%)$ was similar in all cases, studies by MS revealed major differences in the composition of the acylcarnitine fraction (see below).

Identification of the acylcarnitines. The combined techniques of FAB-MS and constant B/E ratio linked scanning were employed to investigate the composition of the acylcarnitine fraction. As shown in Figure $3 A$, the FAB mass spectrum of a postcarnitine urine sample from patient R-1 revealed major ions at $\mathrm{m} / \mathrm{z} 260,286,288$, and 314 . These four ions are consistent with the expected masses of protonated molecular ions $\left(\mathrm{MH}^{+}\right)$ for hexanoylcarnitine, octenoylcarnitine, octanoylcarnitine, and decenoylcarnitine. Of particular note is the absence of the $\mathrm{m} / \mathrm{z}$ 204 ion, the $\mathrm{MH}^{+}$ion of acetylcarnitine, which is the predominant acylcarnitine observed in normal individuals. The $\mathrm{m} / \mathrm{z} 288$ ion was always seen in the purified urine of the patients with MCAD even prior to carnitine therapy and when on dietary fat restriction. At those times, the organic acid profiles were relatively unremarkable (Fig. $1 D$ ). Further evidence that the $\mathrm{m} / \mathrm{z}$ 288,314 , and 260 ions represent acylcarnitines is provided by their fragmentation patterns, obtained by constant $\mathrm{B} / \mathrm{E}$ ratio linked scanning, in which the characteristic loss of 59 daltons occurs (Fig. $3 B-D$ ). This loss reflects elimination of the trimethylamine, $\left(\mathrm{CH}_{3}\right)_{3} \mathrm{~N}$, fragment of the carnitine molecule (21). The fragmentation pattern of the synthetic octanoylcarnitine $\left(\mathrm{MH}^{+}\right)$ion (Fig. $3 E$ ) is indistinguishable from that of the m/z 288 ion in the patient's urine (Fig. $3 B$ ). Octanoylcarnitine was detectable by this technique in samples originally containing as little as $30-50 \mathrm{nmol}$ (total) acylcarnitine, as determined by radioenzymatic assay. It is noteworthy that the pattern of the acylcarnitine signals, characterized by the relative abundances of the four major ions, was essentially similar in all the MCAD urine extracts, before and after L-carnitine supplementation. In addition, when the acylcarnitine concentration exceeded 300 $\mathrm{nmol} / \mathrm{ml}$, which it did from about 2-6 h following a $100 \mathrm{mg} / \mathrm{kg}$ bolus of carnitine, the acylcarnitine signals were detectable by direct FAB-MS analysis of the urine without purification.

Further confirmation of these acylcarnitine structures was obtained by mild alkaline hydrolysis of the purified acylcarnitine fraction, followed by GC/MS analysis of the liberated acids as their methyl esters. This analysis identified hexanoic, octenoic, octanoic (mainly), and decenoic acids in approximately the same relative concentration as indicated by the $\left(\mathrm{MH}^{+}\right)$ion ratios in the expanded FAB-MS spectrum (Fig. 4). These liberated acids were not seen in the ether extract of the purified acylcarnitine fraction before hydrolysis, or in hydrolysates from the control urines. Acylglycines were completely inert to the hydrolysis conditions. By adding to selected purified urine samples a known amount of $n$-valeric acid, then carrying out base hydrolysis (GC/ MS as described), the approximate relative molar concentrations of the liberated hexanoic and octanoic acids were calculated. The sum of these values, after correction for losses during the purification, was in good agreement with the total acylcarnitine concentration determined radioenzymatically for each urine sample. From the relative abundances of these GC peaks, the proportion of each acylcarnitine in the urine samples of patients R- 1 and $\mathrm{K}$ (Table 1) was estimated as follows: hexanoylcarnitine-13\%, octenoylcarnitine-11\%, octanoylcarnitine-63\%, decenoylcarnitine- $13 \%$. These data support the application of ion-exchange column purification, mild alkaline hydrolysis, and GC as a valid procedure for acylcarnitine analysis, at least in the recognition of metabolic disorders such as MCAD. Bieber and Lewin (27) suggested a similar protocol, but have been unable to offer verification in the absence of a specific method for the intact acylcarnitines.

The mass spectra of the liberated unsaturated acid methyl esters were inconsistent with the double-bond location at position 2 (large $\mathrm{m} / \mathrm{z} 74$ to 87 ratio, absence of $\mathrm{m} / \mathrm{z} 131$ ). Thus, the occurrence of octenoylcarnitine and decenoylcarnitine is not due to a problem associated with the enoyl hydratase of $\beta$-oxidation. 
MCAD URINE (FAB)

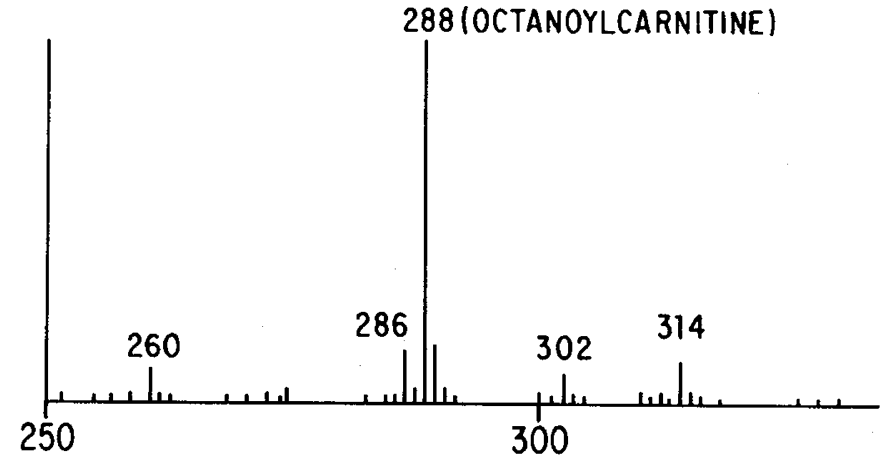

BASE HYDROLYSATE (GC/MS)

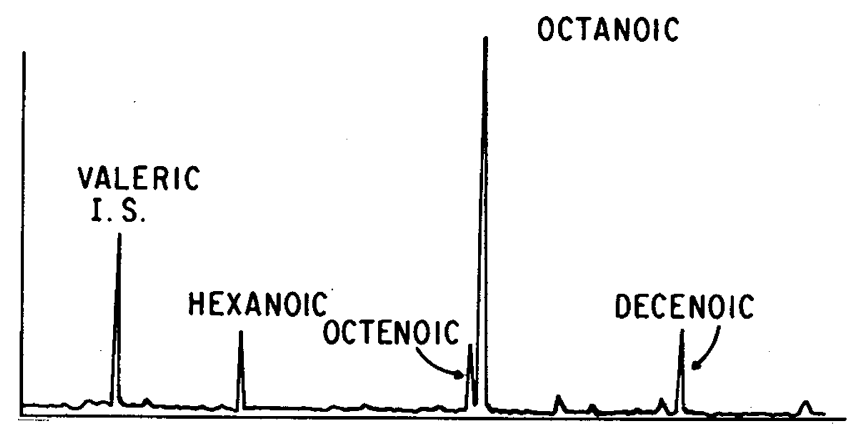

Fig. 4. Analysis of acylcarnitines in MCAD urine by FAB-MS and hydrolysis-GC/MS of liberated acids. $A$, expanded region of FAB-MS spectrum from $\mathrm{R}-1$ showing $\mathrm{MH}^{+}$ions due to acylcarnitines-hexanoyl(m/z 260), octenoyl-(m/z 286), octanoyl-(m/z 288), and decenoyl-(m/z 314). $B$, salient region of GC/MS total ion-current profile for acids liberated from purified acylcarnitine fraction by alkaline hydrolysis. Components were identified and quantified as methyl esters. $\mathrm{N}$-valeric acid was added to the hydrolysate as an internal standard. The major component, octanoic acid, accounted for $63 \%$ of the total liberated acid fraction. Acetic acid is not detectable by this method. Note: the $\mathrm{m} / \mathrm{z} 302$ ion does not appear to represent an acylcarnitine either by B/E linked scanning or base hydrolysis.

More likely, their origin is unsaturated long-chain fatty acid from triglycerides.

Similar analyses for identification of acylcarnitines have been carried out on urine samples from patients $\mathrm{M}-1$ and B-2 reported by Stanley et al. (2), patient TB reported by Chalmers et al. (11), and patient JG reported by Gregersen et al. (9) as well as patients with Reye syndrome and dicarboxylic aciduria secondary to nutritional factors. FAB-MS confirms that the medium-chain acylcarnitines, with octanoylcarnitine being most prominent, are characteristic of the medium-chain defect and include patients TB and JG. These acylcarnitine species have not been observed in any other disorder, including the long-chain acyl-coA dehydrogenase deficiency (two patients; Roe CR, Millington DS, unpublished results). Instead, mostly acetylcarnitine was observed. Long-chain acylcarnitines were tentatively identified in one of the long-chain acyl-coA dehydrogenase deficiency patients after a carnitine load, but it is stressed that these data are preliminary and more research is required before definitive profiles can be published. The lack of octanoylcarnitine was, however, established conclusively.

\section{DISCUSSION}

Gregersen et al. (9) published one of the earliest descriptions of a clinical syndrome characterized by unexplained episodes of lethargy and coma associated with hypoglycemia. These authors provided the first identification of suberylglycine in the urine of that patient (JG), who was only mildly ketotic even when fasted. Unfortunately, the patient expired during a diagnostic fast.

Subsequently, Truscott et al. (10) and Chalmers et al. (11) reported patients with similar clinical presentations. Truscott $e t$ al. (10) demonstrated the occurrence of dicarboxylic aciduria while fasting and noted significant quantities of suberylglycine in the urine. Chalmers et al. (11) reported the occurrence of a Reye-like illness in twin siblings with dicarboxylic aciduria and identified 5-hydroxyhexanoate in the urine of the survivor (patient TB). It was then thought that a chemical toxin, a precursor of 5-hydroxyhexanoate, might have been the precipitating agent.

The likelihood of a defect in the medium chain acyl Co-A dehydrogenase was reported by Kolvraa et al. (4). Unfortunately, fibroblasts were not available for study from patient JG and the enzyme defect in this patient and TB has not been identified directly. Recently, Stanley et al. (2) reported three children in two families with similar clinical course and successfully identified a deficiency of the medium-chain acyl-coA dehydrogenase in each of them. The availability of new assays for this enzyme activity has enabled verification of several more cases (5-8).

The two new patients (R-1 and $K$ ) studied in the present investigation were shown to have deficiency of the medium chain acyl-coA dehydrogenase. Additionally, samples of urine from patients $\mathrm{M}-1, \mathrm{~B}-2, \mathrm{~TB}$, and JG were made available for further evaluation. The organic acid analyses essentially confirmed previous findings of dicarboxylic aciduria (adipate, suberate, sebacate) and revealed the presence of 5-hydroxyhexanoate, hexanoylglycine, and suberylglycine in each. Suberylglycine was clearly present in patient TB which was first examined prior to the availability of the mass spectrum of this compound. The organic aciduria varies with the clinical state. When the patient is ill, it is characteristic and believed to be unique to this disorder (Fig. 1C), since other dicarboxylic aciduria profiles are not associated with hexanoylglycine or suberylglycine. The abnormal metabolites disappear when the patient is under good control. These findings are entirely consistent with those of Divry et al. (17) and Duran et al. (16). Furthermore, dicarboxylic aciduria is not in itself an index of MCAD, as implied in some earlier reports $(3,6,14)$, rather it represents the general condition of defective or compromised mitochondrial $\beta$-oxidation. This condition frequently results from enhanced lipolysis, as many occur in response to infection, when there is an underlying insufficiency of carnitine. Patients with inherited disorders of fat and amino acid metabolism are generally chronically deficient in carnitine (28). The condition can also arise, as documented here and elsewhere (17), during periods of excessive dietary medium-chain triglyceride intake.

The rather specific association of these acylglycines with the MCAD deficiency would suggest that the patient recently reported by Rocchiccioli et al. (25) who had a "Reye-like" illness with hypoglycemia and suberylglycinuria probably also represents this disorder.

Stanley et al. (2) reported decreased plasma free carnitine and increased short-chain acylcarnitines in their patients. They also demonstrated a significant reduction in total carnitine in liver and muscle tissue. The new patients in our study had reduced plasma free carnitine and the ratio of urinary acylcarnitines to urinary free carnitine was significantly elevated in patient R-1 but not in patient $\mathrm{K}$, who was already on dietary fat restriction. These patients responded to carnitine supplementation by increased output of acylcarnitine but not as impressively as the controls. The ratio of urinary acylcarnitine to total carnitine excretion was in the control range of about $25-30 \%$. This is very different from patients with propionic acidemia (29), methylmalonic aciduria (30), and isovaleric acidemia (31) where this ratio ranges from $71-85 \%$.

FAB-MS has revealed that the profile of acylcarnitines excreted in MCAD is unique. The controls, the patients with Reye syndrome and those with diet-induced dicarboxylic aciduria excreted predominantly acetylcarnitine. Acetylcarnitine was not, how- 
ever, seen in patients with MCAD. Instead, our patient's samples and those of Stanley, Chalmers, and Gregersen were characterized by the unequivocal identification of octanoylcarnitine, hexanoylcarnitine, octenoylcarnitine, and decenoylcarnitine. Octanoylcarnitine was clearly present in the largest amount (about $63 \%$ of the total) and the relative abundances of the other acylcarnitines were remarkably similar in all the samples. Duran et al. $(15,16)$ have previously identified octanoylcarnitine in MCAD patients' urine using a less specific paper chromatographic technique. Interestingly, Gregerson et al. (14) observed significantly higher levels of octanoate in the hydrolyzed urine of their patient than could be accounted for by the amounts of the free acids and its glycine conjugate before hydrolysis. This discrepancy has subsequently been ascribed to the carnitine conjugate $(16,17)$, which is now confirmed.

Octanoylcarnitine was always present in the urine of our MCAD patients, regardless of the clinical state. Therefore, even when the organic aciduria is not expressed dramatically, MCAD can be distinguished from Reye syndrome and other dicarboxylic acidurias including the long chain acyl-coA dehydrogenase deficiency. This is particularly important for diagnostic evaluation, since fasting a patient, with the risk of life-threatening consequences, now seems unnecessary. Identification of the acylcarnitine will readily identify the defect. Furthermore, administration of L-carnitine as a bolus enhances the excretion of these diagnostic acylcarnitines, which can now be recognized by a simplified procedure employing mild alkaline hydrolysis and gas chromatographic analysis of the liberated acids, a technique available to most clinical service laboratories.

The clinical similarity between the MCAD and Reye syndrome was recognized before the identification of the enzyme defect, evidenced by the descriptive phrase "Reye-like" syndrome. Where autopsy information is available on children dying suddenly with this defect, cerebral edema and fat accumulation in the liver are observed, as in Reye syndrome. Mamunes et al. (32) have reported elevated plasma octanoate levels in children with Reye syndrome. From this initial observation, the octanoate infusion animal model of Reye syndrome evolved (33) and persists despite the fact that more recent studies have failed to detect octanoate in plasma from Reye syndrome patients (34). However, this animal model appears to be more specific for human MCAD. Recent studies by Kim et al. (35) demonstrate that octanoate produces extensive ultrastructural and functional damage to the mitochondria of the choroid plexus. Neither octanoylcarnitine nor L-carnitine produce such ultrastructural abnormalities in this tissue. These observations may help to explain the findings of Waber et al. (19) who demonstrated that a child with MCAD was able to tolerate fasting without the usual response of hypoglycemia and depressed sensorium following carnitine supplementation.

Our studies demonstrate that the carnitine octanoyltransferase is operational in this disease and can be successfully used to convert medium-chain acyl-coA compounds to nontoxic acylcarnitines. These compounds are then excreted in the urine releasing free coA for other metabolic reactions.

In summary, MCAD is a Reye-like illness in clinical presentation, further characterized by suberylglycinuria, octanoylcarnitinuria, and carnitine deficiency. The evidence also suggests mitochondrial accumulation of toxic medium-chain acyl-coA compounds. L-Carnitine administration, previously shown to protect from hypoglycemia during fasting, enhances the excretion of the acyl-coA compounds as specific medium-chain acylcarnitines. We therefore recommend that treatment of MCAD consist of a combination of reduced dietary fat intake and Lcarnitine supplementation.

Acknowledgments. The authors are grateful to Dr. Charles Stanley, Children's Hospital of Philadelphia for samples from patients $\mathrm{M}-1$ and B-2, Dr. Paul Coates of the same center for documentation of medium-chain acyl-coA deficiency in fibro- blasts from our patients R-1 and $K$. The sample from JG was kindly supplied to Dr. R. A. Chalmers by Dr. N. Gregerson, University of Aarhus, Denmark. We also thank Sigma Tau, Rome, Italy for a generous supply of L-carnitine and Ms. D. Gale and Ms.W. Boone for excellent technical assistance.

\section{REFERENCES}

1. Roe CR, Millington DS, Maltby DA, Bohan TP, Hoppel CL 1983 Status and function of L-carnitine in Reye Syndrome and related metabolic disorders. J Natl Reye Syndrome Found 4:58-59

2. Stanley CA, Hale DE, Coates PM, Hall CL, Corkey BE, Yang W, Kelley RI, Gonzales EL, Williamson JR, Baker L 1983 Medium chain acyl-coA dehydrogenase deficiency in children with non-ketotic hypoglycemia and low carnitine levels. Pediatr Res 17:877-884

3. Gregerson N 1984 Fatty acyl-coA dehydrogenase deficiency: enzyme measurement and studies on alternative metabolism. J Inherited Metab Dis 7(supp 1):28-32

4. Kolvraa S, Gregerson N, Christensen E, Hobolth $\mathrm{N} 1982$ In vitro fibroblast studies in a patient with $\mathrm{C}_{6}-\mathrm{C}_{10}$ dicarboxylic aciduria: evidence for a defect in general acyl-coA dehydrogenase. Clin Chim Acta 126:53-67

5. Coates PM, Stanley CA, Hale DE, Corkey BE, Hall CL, Cortner JA 1982 Fatty acid oxidation in fibroblasts of patients with medium chain acyl-coA dehydrogenase deficiency. Am J Hum Genet 34:48A

6. Rhead WJ, Amendt BA, Fritchman KS, Felts SJ 1983 Dicarboxylic aciduria: deficient $\left(1-{ }^{14} \mathrm{C}\right)$ octanoate oxidation and medium chain acyl-coA dehydrogenase in fibroblasts. Science 221:73-75

7. Divry P, David M, Gregersen N, Kolvraa S, Christensen E, Collett JP, Dellamonica C, Cotte J 1983 Dicarboxylic aciduria due to medium chain acylcoA dehydrogenase defect: A cause of hypoglycemia in childhood. Acta Pediatr Scand 72:943-949

8. Coates PM, Hale DE, Katz MR, Stanley CA, Hall CL 1983 Detection of medium-chain acyl-coA dehydrogenase deficiency in leucocytes. Pediatr Res 17:288A

9. Gregersen N, Lauritzen R, Rasmussen K 1976 Suberylglycine excretion in the urine from a patient with dicarboxylic aciduria. Clin Chim Acta 70:417-425

10. Truscott RJW, Hick L, Pullin C, Halpern B, Wilcken B, Griffiths H, Silink M, Kilham H, Grunseit F 1979 Dicarboxylic aciduria: the response to fasting. Clin Chim Acta 94:31-39

11. Chalmers RA, Lawson AM, Whitelaw A, Purkiss P 1980 Twin siblings with a Reye-like syndrome associated with an abnormal organic aciduria, hypoglycemia, diarrhea, and vomiting with close similarities to Jamaican Vomiting Sickness. Pediatr Res 14:1097-1103

12. Yang W, Roth KS, Coates PB 1982 Hypoglycemic, hypoketotic dicarboxylic aciduria. A possible defect in fatty acid oxidation. Pediatr Res 16:267A

13. Colle E, Mamer OA, Montgomery JA, Miller JD 1983 Episodic hypoglycemia with psi-hydroxy fatty acid excretion. Pediatr Res 17:171-176

14. Gregersen N, Kolvraa S, Rasmussen K, Mortensen PB, Divry P, David M, Hobolth $\mathrm{N} 1983$ General (medium chain) acyl-coA dehydrogenase deficiency (non-ketotic dicarboxylic aciduria): quantitative urinary excretion pattern of 23 biological significant organic acids in 3 cases. Clin Chim Acta 132:181191

15. Duran M, De Klerk JBC, Van Pelt J, Wadman SK, Scholte HR, Beekman RP, Jennekens FG 1983 The analysis of plasma and urinary organic acids during prolonged fasting differentiates between systemic carnitine deficiency and a defect of fatty acid oxidation. J Inher Metab Dis 6(suppl 2):121-122

16. Duran M, De Klerk HBC, Wadman SK, Bruinvis L, Ketting D 1984 The differential diagnosis of dicarboxylic aciduria. J Inherited Metab Dis 7(suppl 1):48-5i

17. Divry P, Vianey-Liaud C, Cotte $J 1984$ Gas chromatography-mass spectrometry (GC/MS) diagnosis of two cases of medium chain acyl-coA dehydrogenase deficiency. J Inherited Metab Dis 7(suppl 1):44-47

18. Del Valle JA, Garcia MJ, Merinero B, Perez-Cerda C, Roman F, Jimenez A Ugarte M, Martinez-Pardo M, Ludena C, Camareno C, del Olmo R, Duran M, Wadman SK 1984 A new patient with dicarboxylic aciduria suggestive of medium chain acyl-coA dehydrogenase deficiency presenting as Reye's syndrome. J Inherited Metab Dis 7:62-64

19. Waber L, Francomono C, Brusilow S, Valle D, Fresman F, Goodman S 1984 Medium chain acyl-coA dehydrogenase (MCD) deficiency. Pediatr Res 18:302A

20. Bohmer T, Bremer J 1968 Propionylcarnitine: physiological variations in vivo. Biochim Biophys Acta 152:559-567

21. Millington DS, Roe CR, Maltby DA 1984 Application of high resolution fast atom bombardment and constant $B / E$ ratio linked scanning to the identification and analysis of acylcarnitines in metabolic disease. Biomed Mass Spectrom 11:236-241

22. Brass EP, Hoppel CL 1978 Carnitine metabolism in the fasting rat. J Biol Chem 253:2688-2693

23. Coates PM, Hale DE, Stanley CA, Corkey BE, Cortner JA Genetic deficiency of medium chain acyl-coA dehydrogenase: studies in cultured skin fibroblasts and peripheral mononuclear leukocytes. Pediatr Res (in press)

24. Frerman FE, Sabran JL, Taylor JL, Grossberg SE 1983 Leucine catabolism during the differentiation of 3T3-L/ cells. Expression of a mitochondrial enzyme system. J Biol Chem 258:7087-7093.

25. Rocchiccioli F, Cartier PH, Bougneres PF 1984 Mass spectrometric identifi- 
cation of abnormal aromatic compounds in the urine of a child with Reye like syndrome. Biomed Mass Spectrom 11:127-131

26. Chalmers RA, Lawson AM 1982 Organic Acids in Man. Chapman and Hall, New York

27. Bieber LL, Lewin LM 1981 Measurement of carnitine and O-acylcarnitines. Methods in Enzymology 72:282-286

28. Engel AG, Rebouche CJ 1984 Carnitine metabolism and inborn errors. J Inher Metab Dis 7(suppl 1):38-43

29. Roe CR, Millington DS, Maltby DA, Bohan TB, Hoppel CL 1984 L-Carnitine enhances excretion of propionyl-coA as propionylcarnitine in propionic acidemia. J Clin Invest 73:1785-1788

30. Roe CR, Hoppel CL, Stacey TE, Chalmers RA, Tracey RM, Millington DS 1983 Metabolic response to carnitine in methylmalonic aciduria: an effective strategy for elimination of propionyl groups. Arch Dis Child 58:916-920
31. Roe CR, Millington DS, Maltby DA, Kahler SG, Bohan TP 1984 L-Carnitine therapy in isovaleric acidemia. J Clin Invest 74:2290-2295.

32. Mamunes D, DeVries CH, Miller CD, David RB 1974 Fatty acid quantitation in Reye syndrome. In: Pollack JD (ed) Reye Syndrome. Grune and Stratton, New York, pp 245-254

33. Trauner DA, Adams H 1981 Intracranial pressure elevations during octanoate infusion in rabbits: An experimental model of Reye syndrome. Pediatr Res 15:1097-1099

34. Ogburn PL, Sharp H, Lloyd-Still JD, Johnson SB, Holman RT 1982 Abnormal polyunsaturated fatty acid patterns of serum lipids in Reye syndrome. Proc Natl Acad Sci USA 79:908-911

35. Kim CS, Dorgan DR, Roe CR 1984 L-Carnitine: therapeutic strategy for metabolic encephalopathy. Brain Res (in press)

\title{
Effects of Hypoxemia on Gastrointestinal Blood Flow and Gastric Emptying in the Newborn Piglet
}

\author{
JOANNE S. SZABO, BARBARA S. STONESTREET, AND WILLIAM OH \\ Department of Pediatrics, Women and Infants Hospital of Rhode Island, Providence 02908 and Brown University \\ Program in Medicine, Providence, Rhode Island 02912
}

\begin{abstract}
The effects of severe hypoxemia on gastrointestinal (GI) blood flow and gastric emptying were studied in nine 2- to 4-day-old piglets which were mechanically ventilated while receiving nitrous oxide anesthesia. Each animal was studied during a control period of oxygenation $\left(\mathrm{PaO}_{2} 91 \pm 8\right.$ torr $), 35 \mathrm{~min}$ of hypoxemia $\left(\mathrm{PaO}_{2} 29 \pm 1\right.$ torr), and a recovery period $\left(\mathrm{PaO}_{2} 90 \pm 5\right.$ torr) (mean \pm SEM). During each study period, the animal received a $10 \%$ dextrose test meal with phenol red marker $(22 \mathrm{ml} /$ $\mathrm{kg}$ ), gastric residual volumes were determined at 10 -min intervals over 30-min study periods using a dye dilution double sampling technique, and GI blood flow (radionuclide-labeled microspheres), $\mathrm{O}_{2}$ delivery, $\mathrm{O}_{2}$ extraction, and $\mathrm{O}_{2}$ consumption were measured at the end of the 30 min period. Hypoxemia resulted in decreased blood flow to the following GI organs: stomach, jejunal and ileal mucosa-submucosa, and colon decreased 62,31 , and $35 \%$, respectively $(p<0.05)$. Jejunal and ileal muscularis blood flow remained unchanged. Oxygen delivery and consumption by GI tract decreased 79 and $58 \%$, respectively; whereas oxygen extraction of GI tract increased $115 \%$. Values returned toward baseline levels during the recovery period. The hypoxemic gastric emptying pattern showed significantly greater gastric residuals at $\mathbf{2 0}$ min compared to the 10 -min value $(p<0.05)$. This pattern was different than that observed during control and recovery periods. We conclude that severe hypoxemia results in decreased GI blood flow, tissue oxygenation, and an altered gastric emptying pattern. These observations may have clinical significance for feeding infants following an hypoxemic episode. (Pediatr Res 19: 466-471, 1985)
\end{abstract}

Received July 9, 1984; accepted January 7, 1985.

Reprint requests to Dr. William Oh, 50 Maude Street, Providence, RI 02908

Supported in part by Training Grant 1 T32HD07232-01 from the National Institute of Child Health and Human Development, Bethesda, MD.

\author{
Abbreviations \\ $\mathrm{CaO}_{2}$, arterial oxygen content \\ $\mathrm{CpvO}_{2}$, portal venous oxygen content \\ $\mathrm{DO}_{2} \mathrm{GI}$, oxygen delivery to gastrointestinal tract \\ $\mathrm{NO}_{2}$, nitrous oxide \\ $\mathrm{O}_{2}$ ExGI, oxygen extraction of the GI tract \\ OG, orogastric \\ VO $\mathrm{O}_{2} \mathrm{GI}$, oxygen consumption by GI tract \\ GI, gastrointestinal
}

Hypoxemia places the neonate at risk for developing GI complications. Among those suggested by clinical studies are necrotizing enterocolitis as a result of perinatal asphyxia and decreased GI blood flow (1), and decreased gastric emptying resulting from respiratory distress syndrome and congenital cyanotic heart disease $(2,3)$

The effect of hypoxemia on GI blood flow appears to vary depending on the degree of hypoxemia and perhaps the age of the subject. Moderate hypoxemia causes intestinal hyperemia in adult animals as a means of maintaining constant $\mathrm{O}_{2}$ delivery and consumption to the GI tract $(4,5)$. Severe hypoxemia in fetal and newborn ruminant animals results, instead, in significant increases in $\mathrm{O}_{2}$ extraction as an attempt to maintain $\mathrm{GI} \mathrm{O}_{2}$ consumption $(6,7)$. The compensatory response utilized by hypoxemic, nonruminant newborn animals to maintain adequate GI tissue oxygenation has not been previously defined.

Hypoxemia and ischemia alter the viability of GI motor function (8). Previous studies in adult animals show an association between GI blood flow and motility (9-13). These studies suggest that changes in blood flow may produce alterations in motility and vice versa. Such an association would be important to verify in the newborn since altered gastrointestinal motility may ac- 